\title{
Toward a Molecular Reorganization Energy-Based Analysis of Third- Order Nonlinear Optical Properties of Polymethine Dyes and J-Aggregates
}

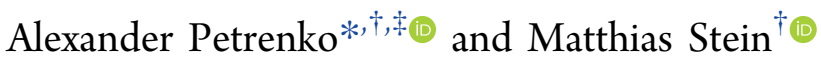 \\ ${ }^{\dagger}$ Molecular Simulations and Design Group, Max Planck Institute for Dynamics of Complex Technical Systems, Sandtorstrasse 1, \\ 39106 Magdeburg, Germany \\ ${ }^{\ddagger}$ Chemistry Department, Pohang University of Science and Technology, Namgu, Pohang 790-784, Korea
}

Supporting Information

\begin{abstract}
This work demonstrates the feasibility and applicability of the theory of extended multiphonon electron transitions for the description of nonlinear optical properties of polymethine dyes using quantum chemistry and model calculations. The transformation of a strong one-photon absorption band in dye monomers to a weak two-photon absorption band is rationalized from the electron-nuclear resonance condition. The power law fitting of the results of quantum chemical computations of nonlinear optical properties allows the predicting of the shift of the corresponding Egorov-like resonance curve to the shortest dye in the vinylogous series of dye monomers. The results presented provide an insight and guide for the rational molecular design and application of polymethine dyes.
\end{abstract}

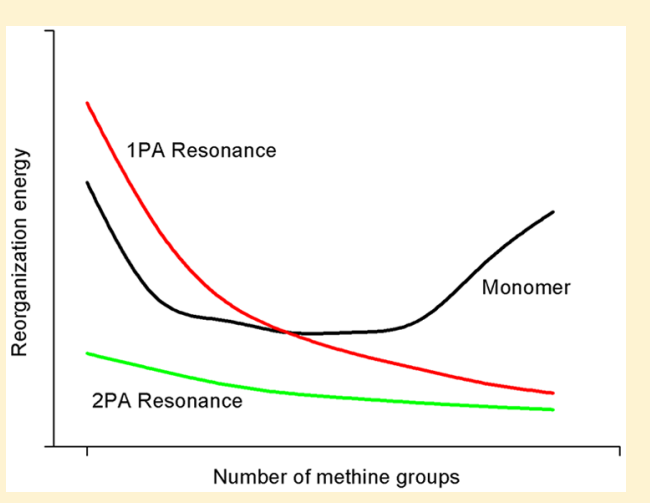

\section{INTRODUCTION}

The concepts of a model spin-Hamiltonian and subsequent model Hamiltonian studies are widespread in the description of magnetic resonance and optical phenomena of molecular and biomolecular systems. ${ }^{1-5}$ The parameters of such a model Hamiltonian (here the spin-Hamiltonian) can be recovered by fitting theoretical curves to experimental spectra. The obtained parameters still require a careful validation and interpretation sometimes augmented by the results of quantum chemical calculations. ${ }^{5-8}$ Quantum chemical calculations allow the establishing of the structural and environmental factors that affect the model Hamiltonian parameters and provide help for a computational design of molecular systems with particular properties for the desired applications. Cyanine-like polymethines are particularly promising organic dyes for many present-day applications including bioimaging ${ }^{9-12}$ and photodynamic therapy. ${ }^{13,14}$ Some of their supramolecular aggregates in solution (J-aggregates) can be detected by the appearance of a narrow and intense absorption band (J-band) which is redshifted with respect to the monomer band. These J-aggregates currently attract increasing interest due to their potential applications in nanoscience. In ref 15 , an attempt of a unified description of linear optical properties of polymethine dyes and their J-aggregates has been made based on the theory of multiphonon electron transitions. ${ }^{16}$ This new approach, the theory of extended multiphonon electron transitions (EMETs), assigns the most intense absorption band in a series of a cyanine dyes and the J-band to an electron nuclear resonance that occurs at a specific length of the polymethine chain. The most important parameter of the EMET theory is the strength of electron nuclear coupling, measured by the nuclear reorganization energy (RE). Our theoretical analysis ${ }^{17}$ is based on the comparison of the quantum chemical curve $\mathrm{RE}(n)$ in a vinylogous series of polymethine dyes, in particularly thiacarbocyanine, thiapyrylocarbocyanine, carbocyanine, and the resonance curve $\operatorname{RE}_{\text {res }}(n)$ is predicted by the EMET theory. Our analysis resolves a long-standing puzzle in the theoretical description of environmental effects on the spectra of carbocyanines and the dependence of the possibility of J-band formation in J-aggregates on the chemical dye structure. In this work, we apply our molecular reorganization energy-based analysis and modeling from the EMET theory to the description of the two-photon absorption property of a special case of polymethine dye, the selenopyrylium polymethine dye. ${ }^{18}$ The position of the Egorov-like resonance curve $\mathrm{RE}_{\text {res } 2}(n)$ for the two-photon absorption is also supported by the results of a power law fitting of the results of our quantum chemical computations of third-order polarizability $(\gamma)$ in the vinylogous series of selenopyrylium as well as thiacyanine and thiapyrylocyanine dyes. Our results allow the proposition of a novel, molecular reorganization energy-based molecular design strategy for cyanine-like polymethine dyes with enhanced two-photon absorption

Received: May 28, 2019

Revised: August 29, 2019

Published: October 7, 2019 
properties for bioimaging and photodynamic therapy applications.

Our main motivation is to demonstrate that the EMET approach, developed for the description of linear optical transitions in polymethine dyes and J-aggregates, can also be applied to the description of nonlinear optical properties of polymethine dyes including their two-photon absorption properties. Quantum chemical calculations and the analysis of parameters entering into the new variant of the EMET theory as well as numerical modeling will be essential to study the possibility of formation of the electron-nuclear resonance state, which then gives rise to the strong two-photon absorption band.

We here compute optical spectra of a series of model polymethine dyes with different parameters from the model Hamiltonian of the EMET theory and compare them with experimental results from one-photon and two-photon spectra of selenopyrylium dye Se-3C whose structural formula is represented (together with other members of the vinylogous series Se-5C and Se-7C) in Figure 1.

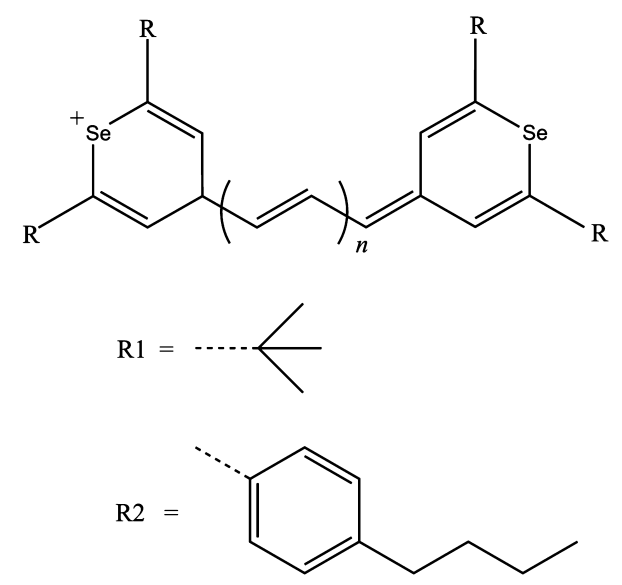

$$
\begin{aligned}
& \text { Se-3C: } n=1, \mathrm{R}=\mathrm{R} 1 \\
& \text { Se-5C: } n=2, \mathrm{R}=\mathrm{R} 2 \\
& \text { Se-7C: } n=3, \mathrm{R}=\mathrm{R} 2
\end{aligned}
$$

Figure 1. Chemical structure of selenopyrylium-terminated polymethine dye cations studied in ref 18 . The monovalent counterion is not shown.

The two-photon optical spectra of the Se-3C dye ${ }^{18}$ are shown in Figure 2. We also compute the third-order polarizability $(\gamma)$-third-order nonlinear optical coefficient in the vinylogous series of thiacyanine and thiapyrylocyanine dyes using a simplified perturbation approach. The obtained results as well as results ${ }^{18}$ from selenopyrylium dyes are fitted with a power law approach in order to calculate the dependence of the effective conjugation length of cyanine chromophore $L(n)$ (sometimes also called the "effective length of cyanine chromophore") on the third-order nonlinear optical parameters. The comparison of computed optical spectra of a model cyanine with the two-photon spectra of selenopyrylium dye Se$3 \mathrm{C}$ and the extracted dependence $L(n)$ of the effective conjugation length for the third-order nonlinear optical parameters then allow us to predict the possible position of

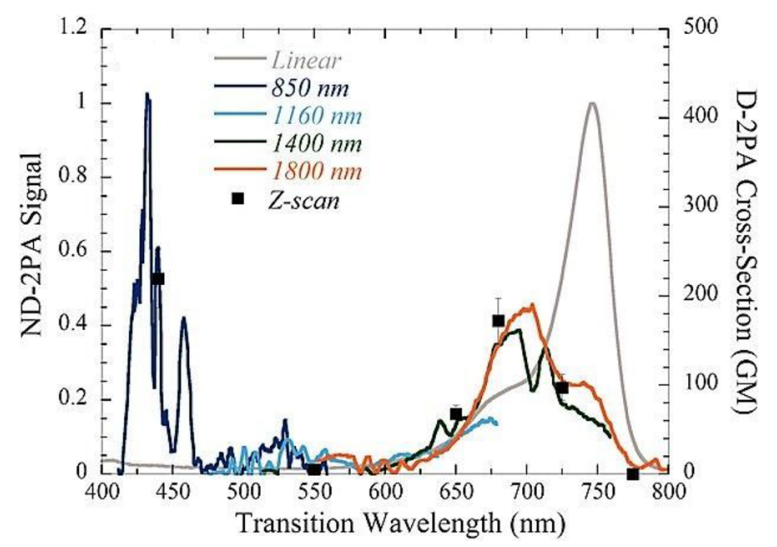

Figure 2. Nondegenerate 2PA spectra for a solution of selenopyrylium-terminated polymethine dye Se-3C dissolved in chloroform. Different pump wavelengths were used to observe the full ND-2PA spectra. Solid circles are degenerate 2PA (D-2PA) cross sections derived from femtosecond-pulsed $Z$-scan measurements. $1 \mathrm{GM}$ is defined as $1 \times 10^{-50} \mathrm{~cm}^{4} \mathrm{~s}$ photon ${ }^{-1}$. Experimental uncertainties in the values were estimated to be $\pm 10 \%$. The linear absorption spectra are shown as reference. Reproduced from Hales et al. ${ }^{18}$ with permission from American Association for the Advancement of Science (AAAS).

an Egorov-like resonance curve $\mathrm{RE}_{\text {res2 }}(n)$ in the (RE, $\left.n\right)$ plane for the two-photon absorption.

The extension of the Egorov theory to the case of twophoton absorption will affect mainly the resulting resonance curve for two-photon absorption. We can show that this transformation is determined by a difference in the Egorov model effective conjugation length for one-photon and twophoton absorption. In order to determine a precise form of resonance from first-principles, the Egorov-like theory has to be extended by using an effective Hamiltonian of the interaction of the system with the light electric field for twophoton absorption. We derive an expression for an Egorov-like new effective Hamiltonian of the interaction of the molecular system with a light electric field for two-photon absorption.

\section{METHODS}

Theory of Optical Spectra from Extended Multiphonon Electron Transitions. We compute and plot the theoretical absorption spectra of model polymethine dyes. The theoretical absorption spectra depend on $\lambda, R, J_{1}$, and two other parameters of EMET theory: the dumping parameter $(\sigma)$ and the energy of an electron in its final state $\left(J_{2}\right)$. Readers may refer to section 3 of ref 15 for a detailed introduction of the formulas and expressions used here. The only difference is in the notation for the dumping parameter. As already mentioned above, the EMET theory of polymethine dyes and J-aggregates is based on the theory of multiphonon electron transitions. Compared to the Hamiltonian of the standard theory of multiphonon electron transitions, ${ }^{16}$ in the EMET theory the Hamiltonian is more complicated and advanced by introducing an additional electronic potential well $V_{2}(\boldsymbol{r}-\boldsymbol{R})$ separated from the initial well $V_{1}(r)$ by the distance $R \equiv|R|$ 


$$
\begin{aligned}
H= & -\frac{\hbar}{2 m} \Delta_{\mathbf{r}}+V_{1}(\boldsymbol{r})+V_{2}(\boldsymbol{r}-\boldsymbol{R})+\sum_{k} W_{k}(\boldsymbol{r}) q_{k} \\
& +\frac{1}{2} \sum_{k} \hbar \omega_{k}\left(q_{k}^{2}-\frac{\partial}{\partial q_{k}^{2}}\right)
\end{aligned}
$$

where $r$ is the radius vector of electron, $q_{k}$ are the real normal phonon coordinates, $\omega_{k}$ are the eigenfrequencies of the normal vibrations, and $k$ is a phonon index. The term

$$
\sum_{k} W_{k}(\boldsymbol{r}) q_{k}
$$

is due to the electron-phonon coupling. The reorganization energy of nuclear vibration is defined in the theory in the following form:

$$
\lambda=\hbar \sum_{k} \omega_{k} \tilde{q}_{k}^{2}
$$

where $\tilde{q}_{k}$ are the normal phonon coordinates corresponding to the equilibrium positions of the nuclei when the electron is in the initial or in the final state, respectively.

Using the methods of classical radiation theory and the Einstein model for nuclear vibrations $\omega_{k}=$ const $\equiv \omega$, the following expression for the light absorption factor (the optical extinction coefficient is proportional to this factor) has been obtained $^{15}$

$$
K=K_{0} \exp W
$$

with

$$
\begin{aligned}
W= & \frac{1}{2} \ln \left(\frac{\omega \tau \sinh \beta_{T}}{4 \pi \cosh t}\right)-\frac{2}{\omega \tau}\left(\cosh \beta_{T}-\frac{\cosh t}{\sinh \beta_{T}}\right) \\
& +\left(\beta_{T}-1\right) \frac{1}{\omega \tau \Theta}-\frac{\sinh \beta_{T}}{4 \omega \tau \Theta^{2} \cosh t}
\end{aligned}
$$

in which $\beta_{T}=\frac{\hbar \omega}{2 k_{\mathrm{B}} T}$, and

$$
\begin{aligned}
& t=\frac{\omega \tau_{\mathrm{e}}}{\theta}\left[\frac{A C+B D}{A^{2}+B^{2}}+\frac{2 \Theta(\Theta-1)}{(\Theta-1)^{2}+\left(\frac{\Theta}{\theta}\right)^{2}}+\frac{\theta_{0}^{2}}{\theta_{0}^{2}+1}\right] \\
& \theta=\frac{R \lambda}{\hbar \sqrt{2 J_{1} / m}}, \Theta=\frac{\lambda}{\Delta}, \theta_{0}=\frac{\lambda}{\sigma}, \tau_{\mathrm{e}}=\frac{R}{\sqrt{2 J_{1} / m}}
\end{aligned}
$$

where $\Delta$ defines the thermal effect energy related to the heat absorption in elementary extended multiphonon transition.
The energy $\hbar \Omega$ of the absorbed photon and thermal effect $\Delta$ are related by the law of conservation of energy:

$$
\hbar \Omega=J_{1}-J_{2}+\Delta
$$

The expressions

$$
\begin{aligned}
& A=A\left(\theta, \Theta, \theta_{0}, \xi\right), B=B\left(\theta, \Theta, \theta_{0}, \xi\right), \\
& C=C\left(\theta, \Theta, \theta_{0}, \xi\right), \quad D=D\left(\theta, \Theta, \theta_{0}, \xi\right)
\end{aligned}
$$

and the factor $K_{0}=K_{0}\left(\theta, \Theta, \theta, \xi, \beta_{T}, t, E, J_{1}, m\right)$ with

$$
\xi=\left(1-\frac{\lambda}{J_{1}}\right)^{1 / 2}
$$

are formulated from elementary functions.

The calculated optical spectra of model polymethine dye are determined by the resonance condition between the extended electronic motion and the nuclear reorganization motion during the electronic transition. According to the EMET approach to the nature of optical transitions in polymethine dyes, the resonance occurs when the characteristic time of extended electronic motion becomes equal to the characteristic time of vibration-like motion of the nuclear reorganization. The time scale $\tau_{\mathrm{n}}$ of nuclear reorganization is defined as $\tau_{\mathrm{n}}=$ $\hbar / \lambda$, where $\lambda$ is the RE. The time scale of the electronic motion $\tau_{\mathrm{e}}$ is defined as $\tau_{\mathrm{e}}=R /\left(J_{1} / 2 m\right)^{-1 / 2}$, where $R, J_{1}$, and $m$ are the effective length of the dye polymethine bridges, the energy of an electron in its initial state, and the mass of an electron, respectively. For linear absorption spectra the effective length $R$ is assigned ${ }^{19}$ to be $2.8(n+2) \AA$, where $n$ is the number of methine groups in the polymethine chain. $J_{1} \equiv J_{1}(n)$ is the experimental energy of an electron in the initial state of the cyanine dye, which depends on the number of monomers $n$. We introduce the resonance parameter $\theta=\tau_{\mathrm{e}} / \tau_{\mathrm{n}}$. The resonance occurs when $\theta=1 / 2$, according to the EMET theory. This condition will be extensively exploited in the discussion section. The theoretical resonance condition $\theta=1$ / 2 determines the functional form of the resonance curve $\mathrm{RE}_{\text {res }}(n)$ in the plane $(\mathrm{RE}, n)$

$$
\mathrm{RE}_{\text {res }}(n)=A /(n+2)
$$

where $A=1.375\left(J_{1}(n) / J_{1}(3)\right)^{1 / 2}$.

The third-order polarizability $(\gamma)$-third-order nonlinear optical coefficient in the vinylogous series of thiacyanine and thiapyrylocyanine dyes was calculated using a simplified perturbation approach. Theoretically, the $\gamma$ values are dominated by the so-called $N$-term in the simplified perturbation theory expression for $\gamma^{18,20,21}$

$$
\begin{aligned}
& \gamma(\omega)=\left[(2.998)^{4} \times 10^{23}\right] \times\left(\frac{1}{5}\right) \times 2 \ldots \\
& \left\{\sum_{e^{\prime}}\left[\frac{M_{\mathrm{ge}^{\prime}}{ }^{2} M_{\mathrm{ee}^{\prime}}{ }^{2}}{\left(\Omega_{\mathrm{ge}}-\hbar \omega\right)\left(\Omega_{\mathrm{ge}^{\prime}}-2 \hbar \omega\right)\left(\Omega_{\mathrm{ge}}-\hbar \omega\right)}+\frac{M_{\mathrm{ge}^{2}}{ }^{2} M_{\mathrm{ee}^{\prime}}{ }^{2}}{\left(\Omega_{\mathrm{ge}}^{*}-\hbar \omega\right)\left(\Omega_{\mathrm{ge}^{\prime}}-2 \hbar \omega\right)\left(\Omega_{\mathrm{ge}}-\hbar \omega\right)}\right]\right. \\
& -\left[\frac{M_{\mathrm{ge}}{ }^{4}}{\left(\Omega_{\mathrm{ge}}-\hbar \omega\right)\left(\Omega_{\mathrm{ge}}-\hbar \omega\right)\left(\Omega_{\mathrm{ge}}-\hbar \omega\right)}+\frac{M_{\mathrm{ge}}{ }^{4}}{\left(\Omega_{\mathrm{ge}}^{*}-\hbar \omega\right)\left(\Omega_{\mathrm{ge}}-\hbar \omega\right)\left(\Omega_{\mathrm{ge}}-\hbar \omega\right)}\right] \\
& \left.+\left[\frac{M_{\mathrm{ge}}{ }^{2}\left(M_{\mathrm{gg}}-M_{\mathrm{ee}}\right)^{2}}{\left(\Omega_{\mathrm{ge}}-\hbar \omega\right)\left(\Omega_{\mathrm{ge}}-2 \hbar \omega\right)\left(\Omega_{\mathrm{ge}}-\hbar \omega\right)}+\frac{M_{\mathrm{ge}}{ }^{2}\left(M_{\mathrm{gg}}-M_{\mathrm{ee}}\right)^{2}}{\left(\Omega_{\mathrm{ge}}^{*}-\hbar \omega\right)\left(\Omega_{\mathrm{ge}}-2 \hbar \omega\right)\left(\Omega_{\mathrm{ge}}-\hbar \omega\right)}\right]\right\}
\end{aligned}
$$

where $g$ denotes the ground state, $e$ and $e^{\prime}$ are the first and higher lying excited states, respectively. $M$ is the transition dipole moment between these states, and $\Omega=(E-i \Gamma)$ with $E$ being the difference in energy between the states, and $\Gamma$ is the 
state dumping. The $N$-term is given by the second line of eq 11. The factor of $1 / 5$ is due to an orientational averaging of $\gamma$, and the prefactor is a conversion factor between the input in MKS units (values for $E, M$, and $\Gamma$ ) and the resulting cgs (esu) units for $\gamma(\omega)$. It should be noted here that, in present work, we did not calculated the $D$ - and T-terms of $\gamma$ (which are given by the first and third line of eq 11 , respectively).

Given the strong dependence of the $N$-term on both the values of transition dipole moment $M_{\mathrm{ge}}$ and the energy difference between the ground and excited state $E_{\mathrm{ge}}\left(\gamma \sim M_{\mathrm{ge}}^{4} /\right.$ $\left.E_{\mathrm{ge}}{ }^{3}\right), \gamma$ is indeed expected to exhibit a very steep power-law dependence on the conjugation length. ${ }^{18}$ We estimated the $N$ term in the vinylogous series of thiacyanine and thiapyrylocyanine from the values of the transition dipole moment and the energy difference between the ground and excited state calculated using GAUSSIAN09 ${ }^{22}$ (CIS and HF option and 631(d,p) basis).

\section{RESULTS AND DISCUSSIONS}

Comparison of Computed Optical Spectra of Model Cyanine with Experimental Two-Photon Spectra of Selenopyrylium Dye Se-3C. We are using Egorov's theoretical formulas from ref 15 to compute and plot the theoretical spectra of model polymethine dyes with different sets of model parameters. We do so in order to allow us to determine a valid pair of sets of parameters (see Figure 3,

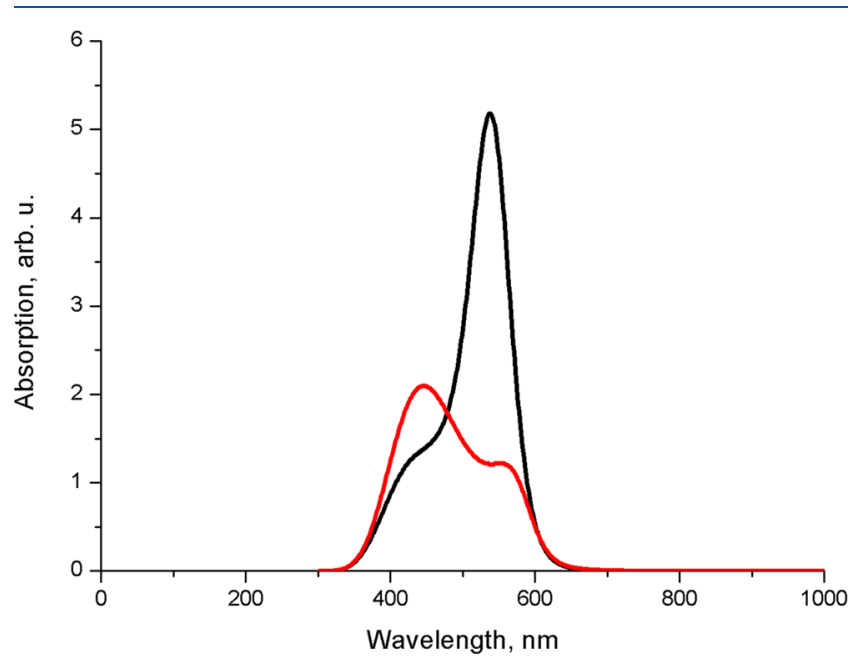

Figure 3. Transformation of the theoretical spectra of a model polymethine dye with different deviations from the resonance condition $\theta=1 / 2$. The black curve describes an optical band corresponding $\theta=0.44$, the red curve describes an optical band corresponding $\theta=0.88$ (shown on different scales). The dumping parameters are taken to be $0.45 \mathrm{eV}$ and $0.34 \mathrm{eV}$, correspondingly. All other simulation parameters are the same as used in ref 1 to reproduce the results of Figure 2 in that work.

caption) that are able to reproduce the experimental transformation from one-photon to the two-photon absorption spectra of selenopyrylium dye Se-3C from ref 18. This is one of the novel issues of this paper which has never been addressed before.

We begin with comparing the results of numerical computations of optical spectra of model cyanines with different values of the resonance parameter $\theta$ of the EMET theory. The resonance parameter can be larger than $1 / 2$ so that the system is in the over-resonance regime. The theoretical absorption band of such a monomer dye consists of two subbands: a low energy L-band and a high energy D-band. ${ }^{15,17}$ The D-band is relatively weak at the condition of the resonance. The deviation of the system from resonance, however, enhances the intensity of the $\mathrm{D}$-band and reduces the L-band. It can clearly be seen from the experimental data of two-photon spectra of selenopyrylium dye Se-3C [see the Zscan data (black squares) and $1800 \mathrm{~nm}$ pump probe measurement results (orange line)] and its linear absorption spectra taken as a reference (see Figure 2) that qualitatively the transformation of spectra from the linear case to the twophoton absorption case can be described in terms of the EMET theory, in particular the redistribution of the intensity of spectra between D- and L-bands. Moreover, the detailed numerical calculations for the model cyanine dye using the formulas from EMET theory (see Figure 3) allow the reproduction of the experimental transformation from the one-photon to the two-photon absorption spectra of the selenopyrylium dye Se-3C quite well. It should be noted here that Egorov's plots from ref 15 actually allow just a qualitative estimate about the possibility of such a transformation.

The origin of the $200 \mathrm{~nm}$ difference between the experimental absorption peak of Se-3C in Figure 2 and the computational peak in Figure 3 is different from the $J_{1}-J_{2}$ values in Se-3C and the model thiacyanine dye used to compute the spectra in Figure 3. It should be also noted here that there is no discussion of the absorption line shapes of Se5C and Se-7C dyes due to the absence of experimental twophoton absorption data in the region of the long wavelength edge of their linear absorption peaks.

Quantum Chemical Calculations of Third-Order Polarizability and Power Law Fitting of Calculated Data. We are computing the third-order polarizability in a vinylogous series of thiacyanine and thiapyrylocyanine molecules and perform a power-law fitting of the calculated data in order to extract the formula for the effective conjugation length for these third-order nonlinear optical parameters that allow us to predict the position of the Egorovlike resonance curve for the two-photon absorption. This approach is novel and has not been treated before.

We estimate the $N$-term in the vinylogous series of thiacyanine and thiapyrylocyanine (Figure 4) from the calculated values of the transition dipole moment and the energy difference between the ground and excited states calculated quantum chemically. The calculated values of the transition dipole moments $M_{\mathrm{ge}}$ and the energy differences between the ground and excited states $E_{\mathrm{ge}}$, as well as calculated static $\gamma$ values, are given in Table 1 . Figure 5 shows the dependence of the $N$-term on the number of methine groups in a particular dye $(n)$. The $N$-term dependencies can be fitted by the $L^{m}$ law $(m=7)$ at small $n$ quite well, but at larger values of $n$ the theoretical values of $\gamma$ increasingly deviate from the power law. It was suggested ${ }^{18}$ that the deviation from the power law happens because the cyanine type dyes are subject to a symmetry breaking at long chain lengths. ${ }^{23-26}$ Although computational studies might provide insight into such a polymethine symmetry breaking, the accuracy of modeling the symmetry breaking process remains challenging. ${ }^{26}$ Many authors follow the view that a symmetry breaking at a long chain length leads to the existence of a so-called "cyanine limit”, manifesting itself in optical spectra. Crossing the cyanine limit at a certain length of the polymethine chain leads to a deep transformation of optical absorption spectra. 
(A)<smiles>C/C=C/c1sc2ccccc2[n+]1CC</smiles>

(B)<smiles>C/C=C/C(C)C=C1C=C(c2ccccc2)SC(c2ccccc2)=C1</smiles><smiles>Cc1ccccc1</smiles>

Figure 4. Thiacyanine (A) and thiapyrylocyanine (B) dye cations with varying lengths of the polymethine chain $(n)$ studied in the present work are shown. The monovalent counterion is not shown.

Table 1. Calculated Transition Energies $\left(E_{\text {ge }}\right.$ in $\left.\mathrm{eV}\right)$, Ground-to-First-Excited-State Transition Dipole Moment $\left(M_{\mathrm{ge}}\right.$ in Debye), and $\left|\gamma_{\mathrm{N}}\right|$ in esu in a Vinylogous Series of Thiacyanine and Thiapyrylocyanine Dyes

\begin{tabular}{rrrr}
$n$ & $E_{\text {ge }}$ & \multicolumn{1}{c}{$M_{\text {ge }}$} & $\left|\gamma_{\mathrm{N}}\right| \times 10^{34}$ \\
& \multicolumn{3}{c}{ Thiacyanine } \\
0 & 5.11 & 9.53 & 0.12 \\
1 & 4.15 & 11.58 & 0.49 \\
2 & 3.68 & 13.99 & 1.49 \\
3 & 3.34 & 16.11 & 3.53 \\
4 & 3.07 & 18.20 & 7.37 \\
5 & 2.90 & 19.18 & 10.79 \\
6 & 3.00 & 18.87 & 9.19 \\
7 & 3.09 & 18.55 & 7.81 \\
& & Thiapyrylocyanine & \\
0 & 3.85 & 13.12 & 1.01 \\
1 & 3.45 & 15.55 & 2.77 \\
2 & 3.01 & 17.83 & 7.23 \\
3 & 2.77 & 19.68 & 13.79 \\
4 & 2.76 & 19.79 & 17.50 \\
5 & 2.86 & 19.15 & 13.82 \\
6 & 2.97 & 18.31 & 8.38 \\
7 & 3.06 & 17.78 & 6.19 \\
\hline
\end{tabular}

The sharp transition characteristic of cyanine spectra undergoes a bathochromic shift $(\sim 100 \mathrm{~nm}$ per additional vinylic unit), followed by a deep modification of the spectra with a large broadening of the band and decrease of its intensity. ${ }^{27}$ Cyanine dyes represent a great challenge for contemporary theoretical chemistry, while in the Egorov approach the unique properties of cyanine dyes are explained by a strong interaction of the cyanine chromophore with condensed media. Therefore, most likely, the deviation of $\gamma(n)$ from the power law is an intrinsic property of the cyanine molecule determined by the peculiarities of quantum transition dynamics of the cyanine molecule in condensed media. Egorov ${ }^{15}$ described the

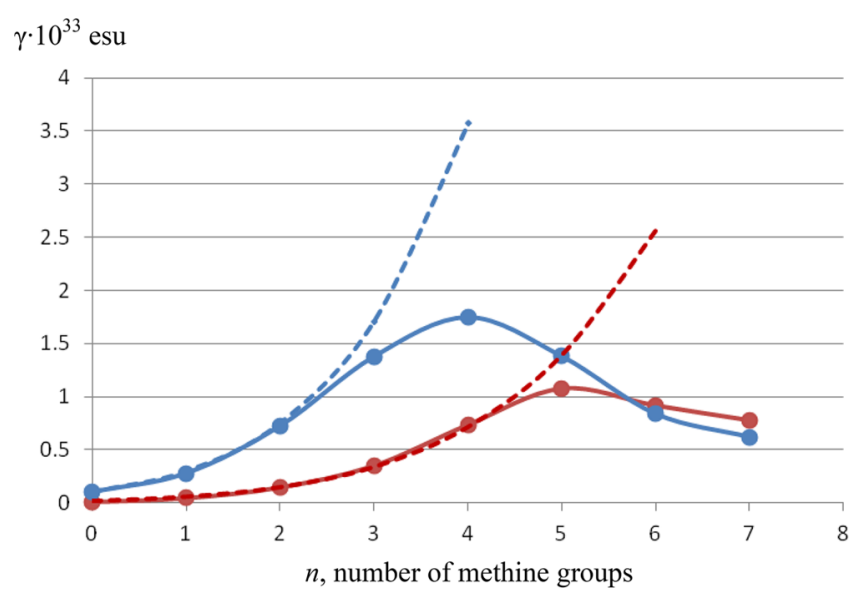

Figure 5. Calculated $\gamma \mathrm{N}$-term dependence in the vinylogous series of thiacyanine (red circles) and thiapyrylocyanine (blue circles). The calculated data were fitted by the $B L^{m}$ law $(m=7)$, where $L=0.28(n$ $+6) \mathrm{nm}$ gives the effective conjugation length $B=0.526 \times 10^{-37} \mathrm{esu} /$ $\mathrm{nm}^{7}$ for thiacyanine and $B=2.65 \times 10^{-37} \mathrm{esu} / \mathrm{nm}^{7}$ for thiapyrylocyanine, respectively.

influence of this dynamics on linear optical absorption in polymethine dyes in terms of the theory of extended multiphonon transitions that is beyond the Born-Oppenheimer and Franck-Condon approximations.

Our method of analysis ${ }^{17}$ which is based on Egorov's theory and quantum chemistry allows the prediction of the redistribution of the optical line shape of cyanine dyes with the solvent and describes the spectra transformation by a gradual extension of the polymethine bridge in a vinylogous series. In the terms of Figure 2 from ref 17, the spectra transformation is explained by an increasing deviation of the dye reorganization energy beyond the electron transfer (ET) resonance values. The dyes at small $n$ corresponding to the beginning of the vinylogous series have a reorganization energy downshifted from the ET resonance reorganization energy which is characterized by the typical cyanine optical line shape. The further increase of $n$ after crossing of the ET resonance line leads to the optical line shape that is intermediate between the regular form of cyanine spectra and "off resonance" spectra. It is one of the essential predictions of Egorov's theory that the "off resonance" long wavelength absorption band is virtually composed of two sub-bands, the L- and D-bands. The D-band is determined by the total reorganization energy, while the Lband is determined by one-half of the global reorganization energy of optical transitions: the relative intensity of these bands depends on the deviation of the system from the electron transfer electron nuclear resonance condition $\theta=1 / 2$. The decrease in the reorganization energy changes the splitting between the L- and D-bands to transform the whole optical band $(\mathrm{L}+\mathrm{D})$ from narrow and intense absorption bands to a slightly asymmetric absorption band (as shown in Figure 2 of Egorov's work ${ }^{15}$ ).

In a recent experimental study ${ }^{18}$ of nonlinear absorption properties in a vinylogous series of selenopyrylium-terminated polymethine dyes (see Figure 1), a high-resolution two-photon absorption spectrum can be obtained (see Figure 2). If we compare this spectrum (together with the linear absorption spectra as reference) with Egorov's theoretical spectra, ${ }^{15}$ some similarity is apparent. Moreover, even more similarity can be noted if we compare the experimental spectra with the results of our simulations based on Egorov's theory presented in 
Figure 3. It shows that Egorov's approach can be applied to the description of two-photon optical spectra of cyanines in a similar approach as to describe the linear optical properties of polymethine dyes. The well-defined techniques of generating polynomials of Krivoglaz and Pekar ${ }^{16,28,29}$ could also be applied analogously. The only difference will be in the effective Hamiltonian of two-photon absorption in comparison with the dipole moment Hamiltonian for the linear absorption case. ${ }^{15,19}$ So far, there is no current construction of an effective Hamiltonian of two-photon absorption in the literature. The existing two-photon absorption calculations are solely based on the "sum over states" formulation of perturbation theory, ${ }^{18,30,31}$ that relies heavily on the Born-Oppenheimer and FranckCondon approximations. The Egorov approach and the results obtained for linear optical properties of polymethine dyes are clearly beyond the $\mathrm{BO}$ and FC approximations.

We present a new effective Hamiltonian of the two-photon absorption (see the Supporting Information for an explicit derivation), with which the corresponding Egorov-like theory can be developed, and a new ET resonance condition similar to condition 11 can be obtained. Because the two-photon spectra are determined by the imaginary part of $\gamma$ (see eq 11 ), some information about the resonance conditions for the twophoton absorption can be obtained from the results of our calculation of the $\gamma \mathrm{N}$-term and the power law fitting of the calculated data (see Table 1 and Figure 5). By the power law fitting $\gamma=B L^{7}$, where $L$ is the dye conjugation length, we obtained the following expression for $L: L=0.28(n+6) \mathrm{nm}$ (see Figure 5, caption), which is valid for both dyes: thiacyanine and thiapyrylocyanine. Moreover, the power law fitting $\gamma=B L^{7}$ results of $\gamma$ computations ${ }^{18}$ with the same conjugation length $L=0.28(n+6) \mathrm{nm}$ work quite well also in a vinylogous series of selenopyrylium-terminated polymethine dyes Se-3C, Se-5C, and Se-7C. On the other hand, Egorov's theory used a conjugation length $L=R=0.28(n+2) \mathrm{nm}$ to describe the linear absorption properties of the vinylogous series of thiacyanine. ${ }^{1}$ Therefore, the expected ET resonance line for the two-photon absorption should be modified in comparison with the ET resonance line for one-photon absorption given by eq 10 . The functional form of the resonance curve $\operatorname{RE}_{\text {res2 }}(n)$ in the plane $(\mathrm{RE}, n)$ is determined by the following:

$$
\mathrm{RE}_{\text {res2 }}(n)=A /(n+6)
$$

where $A=1.375\left(J_{1}(n) / J_{1}(3)\right)^{1 / 2}$.

In Figure 6, the two ET resonance lines are compared with the calculated reorganization energy for the vinylogous series of thiacyanines. We can clearly see that for $n=1$ the thiacyanine dye reorganization energy lies above the twophoton resonance line. In Figure 6, we can also see that reorganization energy of a thiapyrylocyanine dye with the same length as a polymethine chain $n=1$ still lies well above the two-photon resonance line which can give rise to a D-band dominance in the long-wavelength two-photon absorption in accordance with our analysis based on Egorov's theory.

The dominance of the D-band manifests itself in the highresolution long-wavelength two-photon absorption of selenopyrylium-terminated polymethine dye Se-3C, corresponding to $n=1$ [see $Z$-scan results (black squares) and $1800 \mathrm{~nm}$ pump probe measurement data (orange line) in Figure 2]. The similarity in third-order NLO properties of selenopyryliumterminated polymethine dyes and thiapyrylium-terminated polymethine dyes was noted above already; therefore, our

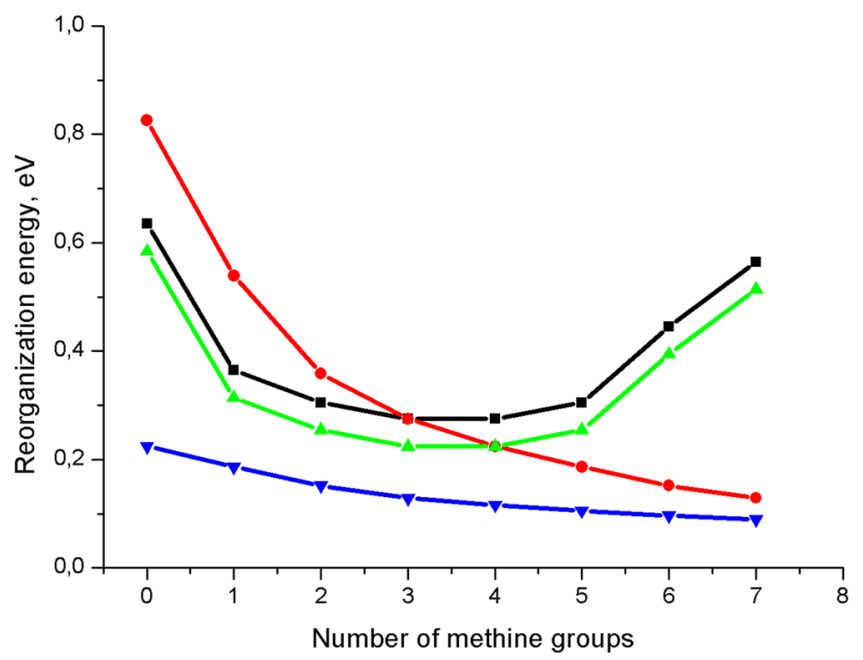

Figure 6. Change of reorganization energy $\lambda\left(S_{0} \rightarrow S_{1}\right)$ in a vinylogous series of thiapyrylocyanine (green triangles) in comparison with thiacyanine (black squares) that approach the expected two-photon ET resonance curve (blue triangles). The reorganization energy required for a one-photon ET resonance is shown as red circles. The solvent reorganization energy $(0.22 \mathrm{eV})$ is taken as a reorganization energy offset.

results for thiapyrylocyanine can be used to interpret the selenopyrylium-terminated polymethine dye data to some extent.

In order to determine a precise form of the two-photon ET resonance curve, the Egorov-like theory has to be further developed by using an effective Hamiltonian of the interaction of the system with the light electric field for the two-photon absorption as outlined in the Supporting Information.

In the end we would like to emphasize that the same Egorov model can be applied to a single cyanine dye and J-aggregates. The only difference is in the numerical values of the parameters.

\section{CONCLUSIONS}

The presented results describe a definite possibility of the extension of the theory of extended multiphonon electron transitions to the description of two-photon optical spectra of polymethine dyes and J-aggregates. The results allow the proposition of a more elaborate molecular design strategy to achieve intense long-wavelength two-photon absorptions of polymethine dyes. This new design approach involves an enhancing of the two-photon absorption of cyanine-like molecules by decreasing the ET reorganization energy of the cyanine molecule (until reaching two-photon ET resonance) by incorporation of additional substituents as terminal groups or directly into the polymethine chain.

\section{ASSOCIATED CONTENT}

\section{S Supporting Information}

The Supporting Information is available free of charge on the ACS Publications website at DOI: 10.1021/acs.jpca.9b05039.

Brief description of the derivation of the simplified Egorov-like expression for an effective Hamiltonian of the interaction of the molecular system with a light electric field for two-photon absorption (PDF) 


\section{AUTHOR INFORMATION}

\section{Corresponding Author}

*E-mail: petrenko@mpi-magdeburg.mpg.de. Phone: +49-3916110-454. Fax: +49-391-6110-403.

\section{ORCID}

Alexander Petrenko: 0000-0002-6661-9864

Matthias Stein: 0000-0001-7793-0052

\section{Notes}

The authors declare no competing financial interest.

\section{ACKNOWLEDGMENTS}

This work was supported in part by the Max-Planck-Society for Advancement of Science and the EU-program ERDF (European Regional Development Fund) of the German Federal State Saxony-Anhalt within the Research Center of Dynamic Systems (CDS).

\section{REFERENCES}

(1) Egorov, V. V.; Alfimov, M. V. Theory of the J-Band: from the Frenkel Exciton to Charge Transfer. Phys.-Usp. 2007, 50, 985-1029.

(2) Eisfeld, A.; Briggs, J. The Shape of the Band of Pseudoisocyanine. Chem. Phys. Lett. 2007, 446, 354-358.

(3) Egorov, V. V. Theory of the J-Band: from the Frenkel Exciton to Charge Transfer. Phys. Procedia 2009, 2, 223-326.

(4) Knapp, E. W. Lineshapes of Molecular Aggregates: Exchange Narrowing and Intersite Correlation. Chem. Phys. 1984, 85, 73-82.

(5) Sinnecker, S.; Neese, F. Theoretical Bioinorganic Spectroscopy. Top. Curr. Chem. 2007, 268, 47-83.

(6) Petrenko, A.; Stein, M. Environment Effects on Spin States, Properties and Dynamics from Multi-level QM/MM Studies. In Spin States in Biochemistry and Inorganic Chemistry; Swart, M., Costas, M., Eds.; Wiley: Chichester, 2016; pp 327-367.

(7) Neese, F. Spin-Hamiltonian Parameters from First Principle Calculations: Theory and Application. In High Resolution EPR Applications to Metalloenzymes; Hanson, G., Berliner, L., Eds.; Springer: New York, 2009; pp 175-229.

(8) Petrenko, A.; Redding, K. Intermolecular Electron Transfer and Exchange Integrals in Photosystem I. Chem. Phys. Lett. 2004, 400, 98-103.

(9) Peng, X.; Wu, T.; Fan, J.; Wang, J.; Zhang, S.; Song, F.; Sun, S. An effective Minor Groove Binder as a Red Fluorescent Marker for Live Cell DNA Imaging and Quantification. Angew. Chem., Int. Ed. 2011, 50, 4180-4183.

(10) James, N. S.; Chen, Y.; Joshi, P.; Ohulchanskyy, T. Y.; Ethirajan, M.; Henary, M.; Strekovsk, L.; Pandey, R. K. Evaluation of Polymethine Dyes as Potential Probes for Near Infrared Fluorescence Imaging of Tumors: Part -1 . Theranostics 2013, 3, 692-702.

(11) Schwechheimer, C.; Römcke, F.; Schepers, U.; Wagenknecht, H.-A. A New Structure - Activity Relationship for Cyanine Dyes to Improve Photostability and Fluorescence Properties for Live Cell Imaging. Chem. Sci. 2018, 9, 6557-6563.

(12) Konig, S. G.; Kramer, R. Accessing Structurally Diverse Near Infrared Cyanine Dyes for Folate Receptor Targeted Cancer Cell Staining. Chem. - Eur. J. 2017, 23, 9306-9312.

(13) Usama, S. M.; Thavornpradit, S.; Burgess, K. Optimized Heptamethine Cyanines for Photodynamic Therapy. ACS Appl. Bio Mater. 2018, 1 (4), 1195-1205.

(14) Atchison, J.; Kamila, S.; Nesbitt, H.; Logan, K. A.; Nicholas, D. H.; Colin, F.; Davis, J.; Callan, B.; McHale, A. P.; Callan, J. F. Iodinated Cyanine Dyes: A New Class of Sensitisers for Use in NIR Activated Photodynamic Therapy (PDT). Chem. Commun. 2017, 53, 2009-2012.

(15) Egorov, V. V. On Electrodynamics of Extended Multiphonon Transitions and Nature of the J-Band. Chem. Phys. 2001, 269, 251283.
(16) Perlin, Yu. E. Modern Methods in the Theory of Many-Phonon Processes. Sov. Phys. Usp. 1964, 6, 542-565.

(17) Petrenko, A.; Stein, M. Molecular Reorganization Energy as a Key Determinant of J-Band Formation in J-Aggregates of Polymethine Dyes. J. Phys. Chem. A 2015, 119, 6773-6780.

(18) Hales, J. M.; Matichak, J.; Barlow, S.; Ohiro, S.; Yesudas, K.; Bredas, J.-L.; Perry, J. W.; Marder, S. R. Design of Polymethine Dyes with Large Third-Order Optical Nonlinearities and Loss Figures of Merit. Science. 2010, 327, 1485-1488.

(19) Egorov, V. V. Nature of the Optical Transition in Polymethine Dyes and J-Aggregates. J. Chem. Phys. 2002, 116, 3090-3103.

(20) Marder, S. R.; Gorman, C. B.; Tiemann, B. G.; Perry, J. W.; Bourhill, G.; Mansour, K. Relation Between Bond-Length Alternation and Second Electronic Hyperpolarizability of Conjugated Organic Molecules. Science 1993, 261, 186-189.

(21) Kogej, T.; Beljonne, D.; Meyers, F.; Perry, J. W.; Marder, S. R.; Bredas, J.-L. Mechanisms for Enhancement of Two-Photon Absorption in Donor-Acceptor Conjugated Chromophores. Chem. Phys. Lett. 1998, 298, 1-6.

(22) Frisch, M. J.; Trucks, G. W.; Schlegel, H. B.; Scuseria, G. E.; Robb, M. A.; Cheeseman, J. R.; Scalmani, G.; Barone, V.; Mennucci, B.; Petersson, G. A.; Nakatsuji, H.; Caricato, M.; Li, X.; Hratchian, H. P.; Izmaylov, A. F.; Bloino, J.; Zheng, G.; Sonnenberg, J. L.; Hada, M.; Ehara, M.; Toyota, K.; Fukuda, R.; Hasegawa, J.; Ishida, M.; Nakajima, T.; Honda, Y.; Kitao, O.; Nakai, H.; Vreven, T.; Montgomery, J. A., Jr.; Peralta, J. E.; Ogliaro, F.; Bearpark, M.; Heyd, J. J.; Brothers, E.; Kudin, K. N.; Staroverov, V. N.; Kobayashi, R.; Normand, J.; Raghavachari, K.; Rendell, A.; Burant, J. C.; Iyengar, S. S.; Tomasi, J.; Cossi, M.; Rega, N.; Millam, J. M.; Klene, M.; Knox, J. E.; Cross, J. B.; Bakken, V.; Adamo, C.; Jaramillo, J.; Gomperts, R.; Stratmann, R. E.; Yazyev, O.; Austin, A. J.; Cammi, R.; Pomelli, C.; Ochterski, J. W.; Martin, R. L.; Morokuma, K.; Zakrzewski, V. G.; Voth, G. A.; Salvador, P.; Dannenberg, J. J.; Dapprich, S.; Daniels, A. D.; Farkas, O.; Foresman, J. B.; Ortiz, J. V.; Cioslowski, J.; Fox, D. J. Gaussian 09; Gaussian, Inc.: Wallingford, CT, 2009.

(23) Tolbert, L. M.; Zhao, X. Beyond the Cyanine Limit: Peierls Distortion and Symmetry Collapse in a Polymetine Dye. J. Am. Chem. Soc. 1997, 119, 3253-3258.

(24) Bouit, P. A.; Aronica, C.; Toupet, L.; Le Guennic, B.; Andraud, C.; Maury, O. Continuous Symmetry Breaking Induced by Ion Pairing Effect in Heptamethine Cyanine Dyes: Beyond the Cyanine Limit. J. Am. Chem. Soc. 2010, 132, 4328-4335.

(25) Yesudas, K. Cationic Cyanine Dyes: Impact of SymmetryBreaking on Optical Absorption and Third-Order Polarizabilities. Phys. Chem. Chem. Phys. 2013, 15, 19465-19477.

(26) Gieseking, R. L.; Ravva, M. K.; Coropceanu, V.; Bredas, J.-L. Benchmarking Density Functional Theory Approaches for the Description of Symmetry Breaking in Long Polymethine Dyes. J. Phys. Chem. C 2016, 120, 9975-9984.

(27) Fabian, J. Symmetry-Lowering Distortion of Near-Infrared Polymethine Dyes - a Study by First-Principles Methods. J. Mol. Struct:: THEOCHEM 2006, 766, 49-60.

(28) Krivoglaz, M. A.; Pekar, S. I. Optical Line Shape of Impurity Absorption and Luminescence in Insulators. Trudy Inst. Fiz. Akad. Nauk Ukr. SSR 1953, 4, 191-207.

(29) Krivoglaz, M. A. Theory of Thermal Transitions. Sov. Phys. JETP 1953, 25, 37-70.

(30) Kamada, K.; Ohta, K.; Iwase, Y.; Kondo, K. Two-Photon Absorption Properties of Symmetric Substituted Diacetylene: Drastic Enhancement of the Cross Section Near One-Photon Absorption Peak. J. Chem. Phys. Lett. 2003, 372, 386-393.

(31) Fu, J.; Padilha, L. A.; Hagan, D. J.; Van Stryland, E. W.; Przhonska, O. V.; Bondar, M. V.; Slominsky, Y. L.; Kachkovski, A. D. Experimental and Theoretical Approaches to Understanding TwoPhoton Absorption Spectra in Polymethine and Squarane Molecules. J. Opt. Soc. Am. B 2007, 24, 67-76. 